Pure and Applied Mathematics Quarterly

Volume 5, Number 2

(Special Issue: In honor of

Friedrich Hirzebruch, Part 1 of 2)

$755-780,2009$

\title{
The Eigencone and Saturation for Spin(8)
}

\author{
Michael Kapovich, Shrawan Kumar and John J. Millson* \\ Dedicated to F. Hirzebruch on the occasion of his eightieth birthday
}

\begin{abstract}
We explicitly calculate the system of restricted triangle inequalities for the group PSO(8) given by Belkale-Kumar, thereby explicitly solving the eigenvalues of a sum problem for this group (equivalently describing the side-lengths of geodesic triangles in the corresponding symmetric space for the metric $d_{\Delta}$ with values in the Weyl chamber $\Delta$ ). We then apply some computer programs to verify the saturation conjecture for the decomposition of tensor products of finite-dimensional irreducible representations of $\operatorname{Spin}(8)$. Namely, we show that for any triple of dominant weights $(\lambda, \mu, \nu)$ such that $\lambda+\mu+\nu$ is in the root lattice, and any positive integer $N$,
\end{abstract}

$$
(V(\lambda) \otimes V(\mu) \otimes V(\nu))^{\operatorname{Spin}(8)} \neq 0
$$

if and only if

$$
(V(N \lambda) \otimes V(N \mu) \otimes V(N \nu))^{\operatorname{Spin}(8)} \neq 0 .
$$

Keywords and phrases: Irreducible representations, Tensor product decomposition.

Received September 10, 2006.

This paper was circulated earlier with the title "Saturation and irredundancy for Spin(8)."

*The authors were partially supported by the NSF individual grants DMS 0405180, DMS 0401084 and DMS 0405606 respectively and together by the FRG grant DMS 0554247 from NSF. 


\section{IntroduCtion}

In this paper we address the following three basic problems in the theory of algebraic groups. The statements of the first two problems and the description of their solution set, the cone $\mathcal{C}(R)$, do not depend on the fundamental group of the underlying group $G$. However, the third problem does depend on the fundamental group of $G$. To pose it in its greatest generality we will assume that $G$ is centerless, see Remark 1.3 below.

Thus, we let $G$ be a connected complex semisimple centerless algebraic group. We fix a Borel subgroup $B$, a maximal torus $T \subset B$ and a maximal compact subgroup $K$. Let $X=G / K$ be the associated symmetric space. Let $\mathfrak{b}, \mathfrak{h}, \mathfrak{k}$ and $\mathfrak{g}$ be the Lie algebras of $B, T, K$ and $G$ respectively. Let $\mathfrak{g}=\mathfrak{k} \oplus \mathfrak{p}$ be the Cartan decomposition. We can and will assume that $\mathfrak{h}$ satisfies $\mathfrak{h}=\mathfrak{h} \cap \mathfrak{k} \oplus \mathfrak{h} \cap \mathfrak{p}$. Let $\mathfrak{a}$ be the second intersection $\mathfrak{h} \cap \mathfrak{p}$ (the Cartan subspace). Let $A$ be the real split subtorus of $T$ corresponding to $\mathfrak{a}$ and $R \subset \mathfrak{a}^{*}$ be the set of roots associated to $(G, T)$. The choice of $B$ determines the set $R^{+} \subset R$ of positive roots and thus the set $\Pi=\left\{\alpha_{1}, \ldots, \alpha_{l}\right\} \subset R^{+}$of simple roots and also the fundamental weights $\left\{\omega_{1}, \ldots, \omega_{l}\right\}, l$ being the rank of $G$. The cone generated by the positive roots determines the dual cone $\Delta \subset \mathfrak{a}$, the (closed) Weyl chamber.

In Section 2 we will introduce the $\Delta$-valued distance $d_{\Delta}$ on the symmetric space $X$. In what follows, by the words "triangle with vertices $x_{1}, x_{2}, x_{3}$ " we will mean the union of the three (unique) oriented geodesic segments (edges) joining each pair of vertices and oriented respectively from $x_{1}$ to $x_{2}, x_{2}$ to $x_{3}$ and $x_{3}$ to $x_{1}$. We then have the following:

Problem 1. The triangle inequalities. Give conditions on a triple $\left(h_{1}, h_{2}, h_{3}\right) \in$ $\Delta^{3}$ that are necessary and sufficient in order that there exists a triangle in $X$ with vertices $x_{1}, x_{2}, x_{3}$ such that $d_{\Delta}\left(x_{1}, x_{2}\right)=h_{1}, d_{\Delta}\left(x_{2}, x_{3}\right)=h_{2}$ and $d_{\Delta}\left(x_{3}, x_{1}\right)=h_{3}$.

Our second problem is the generalization (to general $G$ ) of the problem of finding the possible eigenvalues of a sum of Hermitian matrices given the eigenvalues of the summands. To formulate this problem, define the map

$$
\pi: \mathfrak{p} / K \rightarrow \Delta
$$

by taking $\pi(x)$ to be the unique point in the intersection of $\Delta$ with the $A d K$-orbit of $x$. 
Problem 2. The generalized eigenvalues of a sum problem. Determine the subset $\mathcal{C}=\mathcal{C}(R) \subset \Delta^{3}$ consisting of triples $\left(h_{1}, h_{2}, h_{3}\right) \in \Delta^{3}$ such that there exists a triple $\left(y_{1}, y_{2}, y_{3}\right) \in \mathfrak{p}^{3}$ for which

$$
y_{1}+y_{2}+y_{3}=0
$$

and $\pi\left(y_{1}\right)=h_{1}, \pi\left(y_{2}\right)=h_{2}, \pi\left(y_{3}\right)=h_{3}$.

It turns out that the sets of solutions to Problems 1 and 2 coincide, see [Kl2], $[\mathrm{AMW}],[\mathrm{EL}],[\mathrm{KLM} 1]$ and $[\mathrm{KLM} 2]$. The common solution set $\mathcal{C}$ is in fact a convex homogeneous polyhedral cone $\mathcal{C}$, [BS], see also [KLM1]. The set $\mathcal{C}$ is described in [BS] with a refinement in [KLM1] by a certain system of homogeneous linear inequalities, the triangle inequalities $\widetilde{T I}(R)$, which is, in general, a redundant system. A smaller system, the restricted triangle inequalities $T I(R)$, was introduced by Belkale and Kumar in [BK]. These two systems of inequalities are based on the cup product, resp. the degenerated cup product, on the cohomology of the generalized Grassmannians $G / P$, where $P \subset G$ are maximal parabolic subgroups.

Belkale and Kumar posed the question if the system $T I(R)$ is irredundant (cf. [BK, Section 1.1]). Subsequent to our present work, it was proved in the affirmative by Ressayre $[R]$.

Remark 1.1. The two systems of inequalities $\widetilde{T I}(R)$ and $T I(R)$ coincide in the case of type A root systems (cf. [BK, Lemma 19]). In this case irredundancy of $T I(R)$ was proven by Knutson, Tao and Woodward in [KTW].

In this paper we explicitly determine the system $T I(R)$ for $R=D_{4}$, that is for groups with Lie algebra $\mathfrak{s o}(8)$.

Theorem 1.2. The system of restricted triangle inequalities $T I\left(D_{4}\right)$ is explicitly given in Section 5 and consists of 294 inequalities. Moreover, by virtue of the computer program CONVEX $[\mathrm{F}]$, the cone $\mathcal{C}$ has 306 facets including 12 facets of $\Delta^{3} \subset \mathfrak{a}^{3}$ (and 81 extremal rays). In particular, the system $T I(R)$ is irredundant.

Our third problem concerns the decomposition of tensor products of finitedimensional irreducible representations of a connected complex semisimple group. The first two problems for $G$ are related to the tensor product decomposition problem for the Langlands' dual $G^{\vee}$ of $G$, see [Sp, pages 3-6] for the definition of $G^{\vee}$. We now explain this change of groups. 
There is a natural correspondence of maximal tori $T$ and $T^{\vee}$ for the two groups $G$ and $G^{\vee}$ such that the dominant coweights (the "integral points" in $\Delta$ ) of $G$ are the dominant weights of $G^{\vee}$ whence the input data for Problem 3 for the case of $G^{\vee}$ is a subset consisting of the "integral points" of the input data for Problems 1 and 2 for $G$.

Remark 1.3. Since the representation ring of the simply-connected form of a group contains the representation rings of the other forms, it is natural to consider the decomposition of tensor products problem for the simply-connected form of the group. Under the Langlands' duality the simply-connected group corresponds to the centerless group. It is for this reason that we have assumed in the first paragraph of this introduction that $G$ is centerless.

Let $P^{\vee}$ be the weight lattice of $G^{\vee}$, i.e., $P^{\vee}$ is the character lattice of the maximal torus $T^{\vee} \subset G^{\vee}$. Then,

$$
\mathcal{D}=\mathcal{D}\left(G^{\vee}\right):=P^{\vee} \cap \Delta
$$

is the set of dominant weights of $G^{\vee}$.

Definition 1.4. We define $\left(\mathcal{D}^{3}\right)^{0}$ to be the subsemigroup of $\mathcal{D}^{3}$ consisting of those triples of dominant weights whose sum is in the root lattice $Q^{\vee}$ of $G^{\vee}$.

Given $\lambda \in \mathcal{D}$, let $V(\lambda)$ denote the irreducible representation of $G^{\vee}$ with dominant weight $\lambda$. We now state our third problem.

Problem 3. Determine the semigroup $\mathcal{R}=\mathcal{R}\left(G^{\vee}\right) \subset \mathcal{D}^{3}$ consisting of triples of dominant weights $(\lambda, \mu, \nu)$ such that

$$
(V(\lambda) \otimes V(\mu) \otimes V(\nu))^{G^{\vee}} \neq 0 .
$$

Then, $\mathcal{R} \subset\left(\mathcal{D}^{3}\right)^{0}$. It is well known that Problems 2 and 3 are related, namely that the semigroup $\mathcal{C}^{0}=\mathcal{C}\left(G^{\vee}\right)^{0}:=\mathcal{C}(R) \cap\left(\mathcal{D}^{3}\right)^{0}$ is the saturation of the semigroup $\mathcal{R}\left(G^{\vee}\right)$ in the semigroup $\left(\mathcal{D}^{3}\right)^{0}$. For a more detailed statement, see Theorem 2.5. The following conjecture was made in [KM2]:

Conjecture 1.5 (Saturation conjecture for simply-laced semisimple groups). Suppose that $G^{\vee}$ is a simply-laced simply-connected complex simple Lie group. Then, the semigroup $\mathcal{R}=\mathcal{R}\left(G^{\vee}\right)$ is saturated in $\left(\mathcal{D}^{3}\right)^{0}$. 
In other words, for any $(\lambda, \mu, \nu) \in\left(\mathcal{D}^{3}\right)^{0}$ and any positive integer $N$,

$$
(N \lambda, N \mu, N \nu) \in \mathcal{R} \Rightarrow(\lambda, \mu, \nu) \in \mathcal{R},
$$

i.e., $\mathcal{R}\left(G^{\vee}\right)=\mathcal{C}\left(G^{\vee}\right)^{0}$. This conjecture is known in the case of type $A$ root systems, this is the saturation theorem of Knutson and Tao [KT], see also [B], [DW], [KM1] for alternative proofs.

We now state our second main theorem.

Theorem 1.6. Let $R=D_{4}$ so that $G^{\vee}=\operatorname{Spin}(8)$. A triple $(\lambda, \mu, \nu) \in\left(\mathcal{D}^{3}\right)^{0}$ satisfies $(\lambda, \mu, \nu) \in \mathcal{R}(\operatorname{Spin}(8))$ if and only if $(\lambda, \mu, \nu) \in \mathcal{C}\left(D_{4}\right)$. Equivalently, the semigroup $\mathcal{R}(\operatorname{Spin}(8))$ is saturated in the semigroup $\left(\mathcal{D}^{3}\right)^{0}$.

In order to prove Theorem 1.6 we use the computer program 4ti2, [HHM], to compute the Hilbert basis of the semigroup $\mathcal{C}(\operatorname{Spin}(8))^{0}$. It turns out that this basis consists of 82 elements (just one more than the number of extremal rays). Moreover, modulo the permutations of the vectors $\lambda, \mu, \nu$ and the action of the automorphisms of the Dynkin diagram of $D_{4}$, there are only 10 different semigroup generators. For each of these generator $\left(\lambda_{i}, \mu_{i}, \nu_{i}\right)$ we verify that

$$
\left(\lambda_{i}, \mu_{i}, \nu_{i}\right) \in \mathcal{R}
$$

by applying the MAPLE package WEYL, [S]. Since $\mathcal{R}$ is a semigroup, it then follows that $\mathcal{C}^{0}=\mathcal{R}$.

By [KLM3, Section 9.4], the previous theorem implies the following saturation theorem for the structure constants of the spherical Hecke algebra of $G=P S O(8)$. Considering $P S O(8)$ as a group scheme $\underline{P S O(8)}$ over $\mathbb{Z}$, let $\mathcal{G}$ be the group of its rational points in a nonarchimedean local field $\mathbb{K}$. Let $\mathcal{O}$ be the ring of integers (elements of multiplicative valuation $\leq 1$ ) of $\mathbb{K}$. We let $\mathcal{K}$ be the group of $\mathcal{O}$-rational points of $P S O(8)$. Let $\mathcal{H}_{\mathcal{G}}$ denote the associated spherical Hecke algebra. We recall that the set of dominant coweights $\mathcal{D}$ of $G$ parameterizes the $\mathcal{K}$-double cosets in $\mathcal{G}$ and that the ring $\mathcal{H}_{\mathcal{G}}$ is free over $\mathbb{Z}$ with basis the characteristic functions $\left\{f_{\lambda}: \lambda \in \mathcal{D}\right\}$. We let $*$ denote the (convolution) product in $\mathcal{H}_{\mathcal{G}}$. We have

Theorem 1.7. Let $G=P S O(8)$. For $\lambda, \mu, \nu \in \mathcal{D}$, the characteristic function of the identity $\mathcal{K}$-double coset occurs in the expansion of the product $f_{\lambda} * f_{\mu} * f_{\nu}$ in terms of the above basis if and only if the triple $(\lambda, \mu, \nu) \in \mathcal{C}$ and $\lambda+\mu+\nu$ is in the coroot lattice $Q^{\vee}$ of $G$. 
We conclude this introduction by noting that the triangle inequalities for the rank two groups were explicitly computed in [KLM1] and the restricted triangle inequalities for the rank three groups were computed in $[\mathrm{KuLM}]$. The semigroups of solutions of the tensor product problem for the rank two cases were computed in [KM2]. There are many examples showing that in the nonsimply-laced case, the semigroup $\mathcal{R}$ is not saturated in $\left(\mathcal{D}^{3}\right)^{0}$. In particular, saturation fails for all nonsimply-laced groups of rank $\leq 4$. On the other hand, by the saturation theorem of Knutson and Tao, Saturation Conjecture 1.5 holds for $G^{\vee}=S L(n, \mathbb{C})$. Thus, the first new test case for Conjecture 1.5 is the case of $G^{\vee}=\operatorname{Spin}(8)$ considered in this paper.

Acknowledgement. We are grateful to the referee for useful remarks and suggestions.

\section{FURTHER DISCUSSION OF THE THREE PROBLEMS}

In this section we give some more details about the three problems formulated in the Introduction. We follow the same notation (as in the Introduction). In particular, $G$ is a connected complex semisimple adjoint (centerless) group (with root system $R$ ) and $G^{\vee}$ is its Langlands' dual, which is simply-connected (since $G$ is adjoint).

2.1. The distance $d_{\Delta}$. We now define the $\Delta$-valued distance $d_{\Delta}$. Let $A_{\Delta}$ be the image of $\Delta$ under the exponential map exp $: \mathfrak{g} \rightarrow G$. We will need the following basic theorem, the Cartan decomposition for the group $G$, see [He, Theorem 1.1, pg. 402].

Theorem 2.1. We have

$$
G=K A_{\Delta} K
$$

Moreover, for any $g \in G$, the intersection of the double coset $K g K$ with $A_{\Delta}$ consists of a single point to be denoted $a(g)$.

Let $\overline{x_{1} x_{2}}$ be the oriented geodesic segment in $X=G / K$ joining the point $x_{1}$ to the point $x_{2}$. Then, there exists an element $g \in G$ which sends $x_{1}$ to the base point $o=e K$ and $x_{2}$ to $y=\exp (\delta)$, where $\delta \in \Delta$. Note that the point $\delta$ is uniquely determined by $\overline{x_{1} x_{2}}$. We define a map $\sigma$ from $G$-orbits of oriented geodesic segments to $\Delta$ by 


$$
\sigma\left(\overline{x_{1} x_{2}}\right)=\delta
$$

Clearly we have the following consequence of the Cartan decomposition.

Lemma 2.2. The map $\sigma$ gives rise to a one-to-one correspondence between the $G$-orbits of oriented geodesic segments in $X$ and the points of $\Delta$.

In the rank 1 case $\sigma\left(\overline{x_{1} x_{2}}\right)$ is just the length of the geodesic segment $\overline{x_{1} x_{2}}$.

Definition 2.3. We call $\sigma\left(\overline{x_{1} x_{2}}\right)$ the $\Delta$-length of $\overline{x_{1} x_{2}}$ or the $\Delta$-distance between $x_{1}$ and $x_{2}$. We write $d_{\Delta}\left(x_{1}, x_{2}\right)=\sigma\left(\overline{x_{1} x_{2}}\right)$.

We note the formula

$$
d_{\Delta}\left(x_{1}, x_{2}\right)=\log a\left(g_{1}^{-1} g_{2}\right) \text { where } x_{1}=g_{1} K, x_{2}=g_{2} K .
$$

Remark 2.4. The delta-distance is symmetric in the sense that

$$
d_{\Delta}\left(x_{1}, x_{2}\right)=-w_{o} d_{\Delta}\left(x_{2}, x_{1}\right),
$$

where $w_{o}$ is the unique longest element in the Weyl group. If $w_{o}=-1$ (in particular, in the case of the type $D_{4}$ root system studied in this paper), then

$$
d_{\Delta}\left(x_{1}, x_{2}\right)=d_{\Delta}\left(x_{2}, x_{1}\right) .
$$

2.2. The relations between Problems $\mathbf{1}, \mathbf{2}$ and 3. In this subsection we expand the discussion in the Introduction concerning the relations between the three problems. We first discuss the relation between Problems 2 and 3.

The (a)-part of the following theorem is standard, see for example the appendix of [KLM3]. The (b)-part follows from Theorem 1.2 of [KLM1], see also Theorem 1.4 of [KLM2] and the paragraph following it. Of course, the (b)-part is clear for the simply-laced groups. So, the only nontrivial case is essentially that of the group $G$ corresponding to the root systems of type $B_{l}$. In this case, Belkale and Kumar have shown that the triangle inequalities themselves coincide under the identification of $\mathfrak{a}$ with $\mathfrak{a}^{*}$ (via any invariant form).

Theorem 2.5. (a) For any semisimple adjoint group $G$ with root system $R$, under the identification of $\mathfrak{a}$ with $\mathfrak{a}^{*}$ (via any invariant form),

$$
\mathcal{R}\left(G^{\vee}\right) \subset \mathcal{C}\left(R^{\vee}\right) .
$$


Conversely, for any triple $(\lambda, \mu, \nu) \in \mathcal{C}\left(R^{\vee}\right) \cap \mathcal{D}^{3}$, there exists a positive integer $N$ such that $(N \lambda, N \mu, N \nu) \in \mathcal{R}\left(G^{\vee}\right)$.

(b) Under the identification of $\mathfrak{a}^{*}$ with $\mathfrak{a}$,

$$
\mathcal{C}(R)=\mathcal{C}\left(R^{\vee}\right)
$$

Thus, combining the two parts, we get the following intrinsic inclusion:

$$
\mathcal{R}\left(G^{\vee}\right) \subset \mathcal{C}(R)
$$

We recall the following standard definition. Suppose that $S_{1} \subset S_{2}$ is an inclusion of semigroups. Then, the saturation of $S_{1}$ in $S_{2}$ is the semigroup of elements $x \in S_{2}$ such that there exists $n \in \mathbb{Z}_{+}$with $n x \in S_{1}$.

Remark 2.6. As a consequence of the previous theorem, the semigroup $\mathcal{C}\left(G^{\vee}\right)^{0}:=$ $\mathcal{C}(R) \cap\left(\mathcal{D}^{3}\right)^{0}$ is the saturation of the semigroup $\mathcal{R}\left(G^{\vee}\right)$ in the semigroup $\left(\mathcal{D}^{3}\right)^{0}$.

We conclude this section by briefly indicating why the solutions of Problems 1 and 2 coincide. First of all, Problem 2 (in the case of an $n$-fold sum) can be reformulated geometrically as a problem of the existence of $n$-gons in $\mathfrak{p}$ with given $\Delta$-valued side-lengths. Both $\mathfrak{p}$ and the symmetric space $X$ admit compactifications by a "visual" sphere $S$ which also has the structure of a spherical building $\partial_{\text {Tits }} X$. The vertices of this building are points in the flag manifolds $G / P$ (where $P$ 's are the maximal parabolic subgroups in $G$ ).

Then, one can define the Gauss map $\Gamma$ which sends the geodesic polygon $\left[x_{1}, \ldots, x_{n}\right] \subset X$ to the weighted configuration

$$
\Gamma\left(\left[x_{1}, \ldots, x_{n}\right]\right)=\left(\left(m_{1}, \xi_{1}\right), \ldots,\left(m_{n}, \xi_{n}\right)\right)
$$

of points in $S$. Here $m_{i}:=d\left(x_{i}, x_{i+1}\right)$ are the ordinary distances, which serve as weights at the points $\xi_{i} \in S\left(x_{n+1}:=x_{1}\right)$. The same definition also works for $X$ replaced by $\mathfrak{p}$. The Gauss map from quadrilaterals in the hyperbolic plane to configurations of four points $\xi_{1}, \xi_{2}, \xi_{3}, \xi_{4}$ on the visual boundary (the circle) is depicted in figure 1. The key problem then is to identify the images of Gauss maps $\Gamma$ for $X$ and $\mathfrak{p}$. It turns out that both consist of "nice" semistable weighted configurations on $S$, where the notion of stability is essentially the one introduced by Mumford in Geometric Invariant Theory (in the case when the weights are natural numbers). We refer the reader to Theorems 5.3 and 5.9 of [KLM1] for the precise statements. 


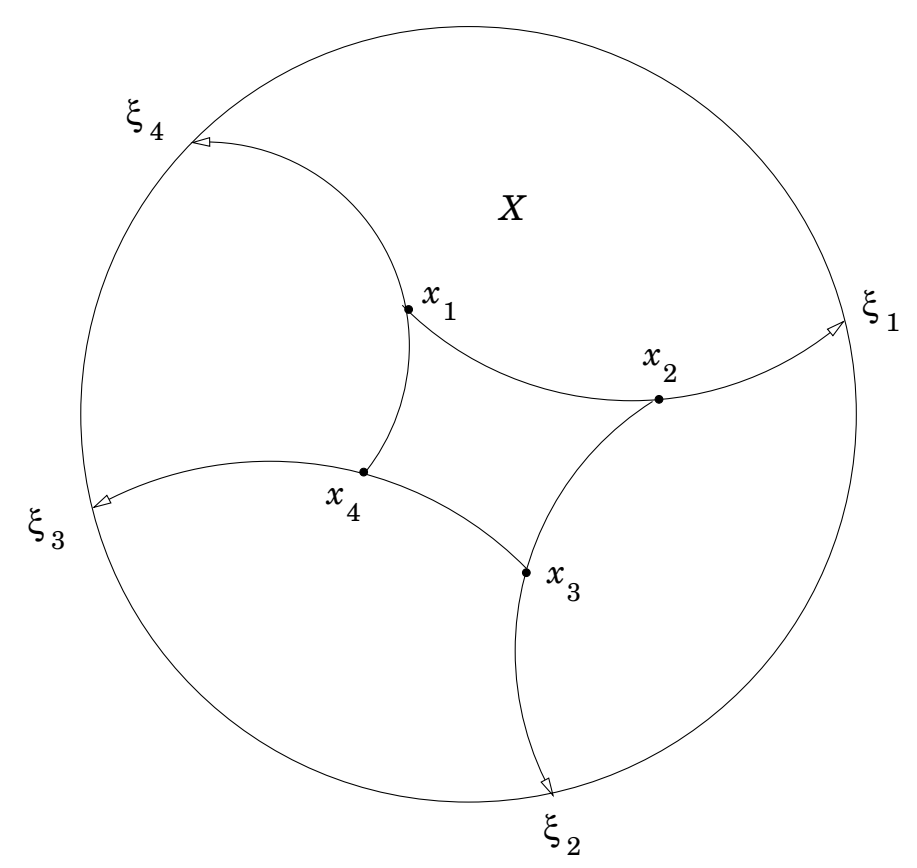

Figure 1. Gauss map.

Therefore, Problems 1 and 2 are both equivalent to the existence problem for semistable weighted configurations on $S$ and hence Problems 1 and 2 are equivalent.

\section{The triangle IneQualities}

We need more notation. We let $S=\left\{s_{1}, \ldots, s_{l}\right\}$ be the set of (simple) reflections in the root hyperplanes defined by the simple roots and let $W \subset$ Aut a be the Weyl group generated by $S$. Then, $W$ can be identified with $N(T) / T, N(T)$ being the normalizer of $T$ in $G$.

Let $\left\{x_{i}\right\}$ be the basis of $\mathfrak{h}$ dual to the basis $\Pi$, i.e., $\alpha_{i}\left(x_{j}\right)=\delta_{i, j}$. We let $\ell$ be the length function on $W$. Let $\alpha_{i}^{\vee}$ be the coroot corresponding to the root $\alpha_{i}$. Also, for a standard parabolic subgroup $P$ of $G$ (i.e., $P \supset B$ ), we let $W_{P} \subset W$ be the subgroup of elements with representatives in $P$ and $W^{P}$ denote the set of shortest length representatives for the cosets $W / W_{P}$ (we recall that each coset has a unique shortest length representative). Let $w_{o}^{P}$ be the unique longest element in $W^{P}$ and $w_{o}$ the longest element of $W$. 
3.1. The triangle inequalities. We now describe the solution of Problem 1 of the Introduction, that is the description of the inequalities determining the $\Delta$-valued side-lengths of geodesic triangles in $X$.

3.1.1. The weak triangle inequalities. We first describe a natural subsystem of the triangle inequalities. The naive triangle inequality

$$
d_{\Delta}\left(x_{1}, x_{3}\right) \leq_{\Delta} d_{\Delta}\left(x_{1}, x_{2}\right)+d_{\Delta}\left(x_{2}, x_{3}\right)
$$

does not hold [KLM3]. Here the order $\leq_{\Delta}$ is the one defined by the (acute) cone $\Delta$. This can be remedied if we replace $\Delta$ by the dual (obtuse) cone $\Delta^{*}$ and let $\leq_{\Delta^{*}}$ denote the associated order. Then, the analogue of the above inequality holds and, in fact, for any element $w$ of the Weyl group $W$, the inequality

$$
w \cdot d_{\Delta}\left(x_{1}, x_{3}\right) \leq_{\Delta^{*}} w \cdot d_{\Delta}\left(x_{1}, x_{2}\right)+d_{\Delta}\left(x_{2}, x_{3}\right)
$$

holds. We call the resulting system of inequalities (as $w$ varies) the weak triangle inequalities to be denoted WTI $(R)$.

For the root systems $R$ of ranks one and two, the weak triangle inequalities already give a solution to Problems 1 and 2 of the Introduction. However, they are no longer sufficient in ranks three or more.

3.1.2. The triangle inequalities. We now describe a system of linear inequalities on $\mathfrak{a}^{3}$ which describes the cone $\mathcal{C}(R)$. However, this system is usually not irredundant. These inequalities (based on the cup-product of Schubert classes) will be called the triangle inequalities. The system of triangle inequalities is independent of the choice of $G$ corresponding to a fixed Lie algebra $\mathfrak{g}$, hence depends only on the root system $R$ associated to $G$. We denote this system of inequalities by $\widetilde{T I}(R)$ or just $\widetilde{T I}$ when the reference to $R$ is clear.

As a consequence of the Bruhat decomposition:

$$
G=\bigsqcup_{w \in W^{P}} B w P
$$

the generalized flag variety $G / P$ is the disjoint union of the subsets

$$
\left\{C_{w}^{P}:=B w P / P\right\}_{w \in W^{P}} .
$$

The subset $C_{w}^{P}$ is biregular isomorphic to the affine space $\mathbb{C}^{\ell(w)}$ and is called a Schubert cell, where $\ell(w)$ is the length of $w$. The closure $X_{w}^{P}$ of $C_{w}^{P}$ is called a Schubert variety. We will use $\left[X_{w}^{P}\right]$ to denote the integral homology class in 
$H_{*}(G / P)$ carried by $X_{w}^{P}$. Then, the integral homology $H_{*}(G / P)$ is a free $\mathbb{Z}^{-}$ module with basis $\left\{\left[X_{w}^{P}\right]: w \in W^{P}\right\}$.

Let $\left\{\epsilon_{w}^{P}: w \in W^{P}\right\}$ denote the dual basis of $H^{*}(G / P)$ under the Kronecker pairing $\langle$,$\rangle between homology and cohomology. Thus, we have for w, w^{\prime} \in W^{P}$,

$$
\left\langle\epsilon_{w}^{P},\left[X_{w^{\prime}}^{P}\right]\right\rangle=\delta_{w, w^{\prime}}
$$

The system of triangle inequalities breaks up into $\operatorname{rank}(\mathfrak{g})$ subsystems $\widetilde{T I}{ }^{P}$, where $P$ runs over standard maximal parabolic subgroups. The subsystem $\widetilde{T I} P$ is controlled by the Schubert calculus in the generalized Grassmannian $G / P$ in the sense that there is one inequality $T_{\mathbf{w}}^{P}$ for each triple of elements $\mathbf{w}=$ $\left(w_{1}, w_{2}, w_{3}\right) \in W^{P}$ such that

$$
\epsilon_{w_{1}}^{P} \cdot \epsilon_{w_{2}}^{P} \cdot \epsilon_{w_{3}}^{P}=\epsilon_{w_{o}^{P}}^{P}
$$

in $H^{*}(G / P)$. To describe the inequality $T_{\mathbf{w}}^{P}$, let $\omega_{P}=\omega_{i_{P}}$ be the fundamental weight corresponding to $P$, where $s_{i_{P}}$ is the unique simple reflection not in $W_{P}$. Then, the standard action of $W$ on $\mathfrak{a}^{*}$ induces a one-to-one correspondence $f$ : $W^{P} \rightarrow W \omega_{P}$ given by $f(w)=w \omega_{P}$. Thus, we may reparametrize the Schubert classes in $G / P$ by the elements of $W^{P} \omega_{P} \subset \mathfrak{a}^{*}$. For $w \in W^{P}$, let $\lambda_{w}=\lambda_{w}^{P}$ denote $w \omega_{P}$; this is called the maximally singular weight corresponding to $w$. Then, the inequality $T_{\mathbf{w}}^{P}$ is given by

$$
\lambda_{w_{1}}\left(-w_{o} h_{1}\right)+\lambda_{w_{2}}\left(-w_{o} h_{2}\right)+\lambda_{w_{3}}\left(-w_{o} h_{3}\right) \geq 0,\left(h_{1}, h_{2}, h_{3}\right) \in \Delta^{3} .
$$

3.2. The restricted triangle inequalities. As we have mentioned earlier, the system of triangle inequalities is in general not an irredundant system. We now describe a subsystem of these inequalities due to Belkale-Kumar.

To this end we recall the definition of the new product $\odot_{0}$ in the cohomology $H^{*}(G / P)$ introduced by Belkale-Kumar [BK, Sect. 6]. We only need to consider the case when $P$ is a standard maximal parabolic subgroup. In this case, we set $x_{P}=x_{i_{P}}$.

Write the cup product in $H^{*}(G / P)$ as follows:

$$
\epsilon_{u}^{P} \cdot \epsilon_{v}^{P}=\sum_{w \in W^{P}} d_{u, v}^{w} \epsilon_{w}^{P} .
$$


Then, by definition,

$$
\epsilon_{u}^{P} \odot_{0} \epsilon_{v}^{P}=\sum_{w \in W^{P}} d_{u, v}^{w} \delta_{u, v}^{w} \epsilon_{w}^{P},
$$

where $\delta_{u, v}^{w}:=1$ if $\left(u^{-1} \rho+v^{-1} \rho-w^{-1} \rho-\rho\right)\left(x_{P}\right)=0$ and $\delta_{u, v}^{w}:=0$ otherwise, where $\rho$ is the (standard) half sum of positive roots of $\mathfrak{g}$.

Recall that $\pi: \mathfrak{p} / K \rightarrow \Delta$ is defined by intersecting an AdK-orbit with $\Delta$. Then, [BK, Theorem 28] gives the following solution of Problem 2 stated in the Introduction:

Theorem 3.1. Let $\left(h_{1}, \ldots, h_{n}\right) \in \Delta^{n}$. Then, the following are equivalent:

(a) There exists $\left(y_{1}, \ldots, y_{n}\right) \in \mathfrak{p}^{n}$ such that $\sum_{j=1}^{n} y_{j}=0$ and $\pi\left(y_{j}\right)=h_{j}$ for all $j=1, \ldots, n$.

(b) For every standard maximal parabolic subgroup $P$ in $G$ and every choice of $n$-tuple $\mathbf{w}=\left(w_{1}, \ldots, w_{n}\right) \in\left(W^{P}\right)^{n}$ such that

$$
\epsilon_{w_{1}}^{P} \odot_{0} \cdots \odot_{0} \epsilon_{w_{n}}^{P}=\epsilon_{w_{o}^{P}}^{P} \in\left(H^{*}(G / P), \odot_{0}\right),
$$

the following inequality holds:

$$
\sum_{j=1}^{n} \lambda_{w_{j}}^{P}\left(-w_{o} h_{j}\right) \geq 0
$$

The collection of inequalities $\left\{T_{\mathbf{w}}^{P}\right\}$, such that $\mathbf{w}$ and $P$ are as in (b), will be referred to as the restricted triangle inequalities.

Remark 3.2. 1. As was the case for $n=3$, the statement in (a) is equivalent to the existence of a geodesic n-gon in $X$ with $\Delta$-side-lengths $h_{1}, h_{2}, \ldots, h_{n}$.

2. As it was noted earlier, for the type $D_{4}$ root system studied in this paper, $w_{o}=-1$ and, hence, the inequalities $\left(T_{\mathbf{w}}^{P}\right)$ simplify to

$$
\sum_{j=1}^{n} \lambda_{w_{j}}^{P}\left(h_{j}\right) \geq 0 .
$$

\section{Determination of the product $\odot_{0}$ in $H^{*}(G / P)$}

From now on, the group $G$ will be taken to be the adjoint group of type $D_{4}$, i.e., $G=P S O(8)$. Since $G$ is simply-laced, the Langlands' dual $G^{\vee}$ has the 
same root system as $G$. However, $G$ being the adjoint group, $G^{\vee}$ is the simplyconnected cover of $G$, i.e., $G^{\vee}=\operatorname{Spin}(8)$. We will only need to consider the maximal parabolic subgroups. We will abbreviate the classes $\epsilon_{w}^{P}$ for $w \in W^{P}$ by $b_{i}^{j}$ according to the following tables. Here the subscript $i$ denotes half of the cohomological degree of $b_{i}^{j}$, i.e., $b_{i}^{j} \in H^{2 i}(G / P)$, and $j$ runs over the indexing set with cardinality equal to the rank of $H^{2 i}(G / P)$. In the case that $H^{2 i}(G / P)$ is of rank one, we suppress the superscript $j$. Moreover, in the following tables, we also list the maximally singular weight $\lambda_{w}:=w \omega_{P}$ associated to the element $w \in W^{P}$ as well as the value $n_{w}:=\left(w^{-1} \rho\right)\left(x_{P}\right)$. We express $\lambda_{w}$ in terms of the standard coordinates $\left\{\epsilon_{i}\right\}_{i=1, \ldots, 4}$ of $\mathfrak{h}^{*}$ as given in [Bo, Planche IV]. We follow the following indexing convention as in loc cit.

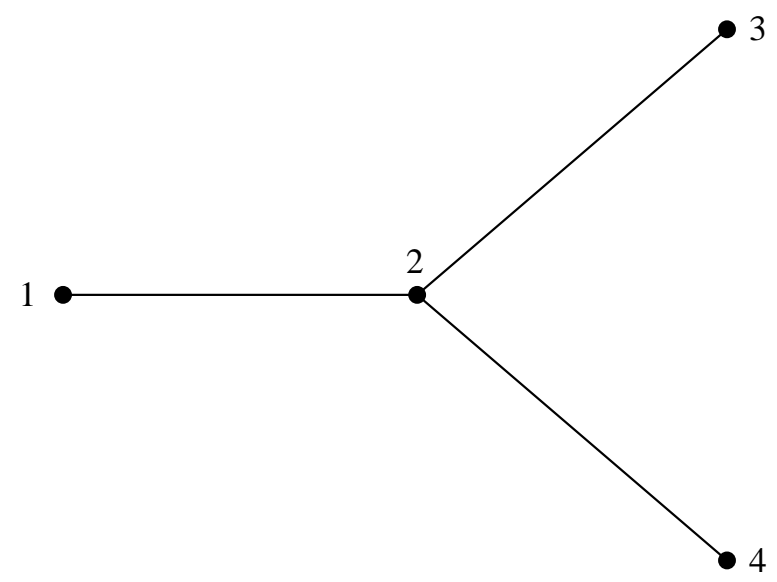

Figure 2. Dynkin diagram for $D_{4}$.

For any $1 \leq i \leq 4$, let $P_{i}$ be the maximal parabolic subgroup of $G$ corresponding to the $i$-th node of the Dynkin diagram, i.e., $W_{P_{i}}$ is generated by the simple reflections $\left\{s_{j} ; j \neq i\right\}$.

To determine the cohomology $H^{*}\left(G / P_{i}\right)$ under the product $\odot_{0}$ for all the maximal parabolic subgroups $P_{i}$, because of the Dynkin automorphisms, we only need to determine it for $i=1,2$. Moreover, since $P_{1}$ is a minuscule parabolic in $G$, the product $\odot_{0}$ coincides with the cup product by [BK, Lemma 19].

In what follows we will use the symbol $F$ to denote the group of automorphisms of the Dynkin diagram of $D_{4}$, so $F$ is isomorphic to the symmetric group $S_{3}$ on the labels $\{1,3,4\}$ of the Dynkin diagram. 
4.1. Determination of $\left(H^{*}\left(G / P_{1}\right), \odot_{0}\right)$. The longest element $w_{o}$ of $W$ is given by

$$
w_{o}=s_{4} s_{2} s_{1} s_{4} s_{2} s_{4} s_{3} s_{2} s_{4} s_{1} s_{2} s_{3},
$$

and it is central in $W$. Moreover, the longest element $w_{o, P_{1}}$ of $W_{P_{1}}$ is given by

$$
w_{o, P_{1}}=s_{3} s_{2} s_{4} s_{3} s_{2} s_{3} .
$$

Thus, the longest element $w_{o}^{P_{1}}$ of $W^{P_{1}}$ is given by (cf. [KuLM, Proposition 2.6])

$$
w_{o}^{P_{1}}=w_{o} w_{o, P_{1}}=s_{1} s_{2} s_{3} s_{4} s_{2} s_{1} .
$$

From this and the fact that $\left|W^{P_{1}}\right|=8$, we see that the elements of $W^{P_{1}}$ are enumerated as in the chart below. To calculate $n_{w}$, use the general formula (cf. [K, Corollary 1.3.22]) for any $w \in W$ :

$$
\rho-w^{-1} \rho=\sum_{\alpha \in R^{+} \cap w^{-1} R^{-}} \alpha,
$$

and for any parabolic subgroup $P$ of $G$ and any $w \in W^{P}$,

$$
R^{+} \cap w^{-1} R^{-} \subset R^{+} \backslash R_{P}^{+},
$$

where $R^{-}:=-R^{+}$and $R_{P}^{+}$is the set of positive roots in the Levi component of $P$. Since $P_{1}$ is a minuscule maximal parabolic subgroup, for any $w \in W^{P_{1}}$, by (4) and (5) we get

$$
\left(\rho-w^{-1} \rho\right)\left(x_{P_{1}}\right)=\ell(w)
$$

From this, the value of $n_{w}$ given in the following chart can easily be verified since $\rho\left(x_{P_{1}}\right)=3$. The value of $\lambda_{w}$ is obtained by explicit calculations.

\begin{tabular}{|c|c|c|c|}
\hline$\epsilon_{w}^{P_{1}}$ & $w$ & $\lambda_{w}$ & $n_{w}$ \\
\hline$b_{0}=1$ & $e$ & $(1,0,0,0)$ & 3 \\
\hline \hline$b_{1}$ & $s_{1}$ & $(0,1,0,0)$ & 2 \\
\hline \hline$b_{2}$ & $s_{2} s_{1}$ & $(0,0,1,0)$ & 1 \\
\hline \hline$b_{3}^{1}$ & $s_{3} s_{2} s_{1}$ & $(0,0,0,1)$ & 0 \\
\hline$b_{3}^{2}$ & $s_{4} s_{2} s_{1}$ & $(0,0,0,-1)$ & 0 \\
\hline \hline$b_{4}$ & $s_{3} s_{4} s_{2} s_{1}$ & $(0,0,-1,0)$ & -1 \\
\hline \hline$b_{5}$ & $s_{2} s_{3} s_{4} s_{2} s_{1}$ & $(0,-1,0,0)$ & -2 \\
\hline \hline$b_{6}$ & $s_{1} s_{2} s_{3} s_{4} s_{2} s_{1}$ & $(-1,0,0,0)$ & -3 \\
\hline
\end{tabular}


Using [KuLM, Lemma 2.9] and the Chevalley formula (cf. [K, Theorem 11.1.7(i)]), all the products in the following table can be determined except the products of $b_{2}$ with $b_{2}$ and $b_{3}^{*}$. Since $b_{1} b_{1}=b_{2}$, using the Chevalley formula twice, we get these products as well.

In the above we have used the symbol $b_{3}^{*}$ to stand for either $b_{3}^{1}$ or $b_{3}^{2}$. In what follows we will use the symbol $b_{i}^{*}$ to denote the class $b_{i}$ with a variable superscript.

Multiplication table for $G / P_{1}$ under the product $\odot_{0}$ :

\begin{tabular}{|c||c||c||c|c|}
\hline$\odot_{0}$ & $b_{1}$ & $b_{2}$ & $b_{3}^{1}$ & $b_{3}^{2}$ \\
\hline \hline$b_{1}$ & $b_{2}$ & & & \\
\hline \hline$b_{2}$ & $b_{3}^{1}+b_{3}^{2}$ & $2 b_{4}$ & & \\
\hline \hline$b_{3}^{1}$ & $b_{4}$ & $b_{5}$ & 0 & \\
\hline$b_{3}^{2}$ & $b_{4}$ & $b_{5}$ & $b_{6}$ & 0 \\
\hline \hline$b_{4}$ & $b_{5}$ & $b_{6}$ & 0 & 0 \\
\hline \hline$b_{5}$ & $b_{6}$ & 0 & 0 & 0 \\
\hline \hline$b_{6}$ & 0 & 0 & 0 & 0 \\
\hline
\end{tabular}

4.2. Determination of $\left(H^{*}\left(G / P_{2}\right), \odot_{0}\right)$. For any parabolic subgroup $P$, let $\theta^{P}$ be the involution of $W^{P}$ defined by

$$
\theta^{P} w=w_{o} w w_{o, P} .
$$

Then, by [KuLM, Section 2.1], $\epsilon_{w}^{P}$ is Poincaré dual to $\epsilon_{\theta^{P} w}^{P}$.

Using (1) and

$$
w_{o, P_{2}}=s_{1} s_{3} s_{4}
$$

we get

$$
w_{o}^{P_{2}}=s_{2} s_{4} s_{1} s_{2} s_{3} s_{2} s_{1} s_{4} s_{2}
$$

The enumeration of $W^{P_{2}}$ as in the following table can be read off from (9) together with the fact that $\left|W^{P_{2}}\right|=24$. The values of $\lambda_{w}$ and $n_{w}$ are obtained by explicit calculations. Observe that the following identities provide some simplification in the calculations of $\lambda_{w}$ and $n_{w}$. 
For any $w \in W^{P_{2}}$,

$$
\lambda_{\theta^{P}(w)}=-\lambda_{w}
$$

and

$$
n_{\theta^{P}(w)}=-n_{w}
$$

In the following table, the two $w^{\prime}$ 's appearing in the same row are $\theta^{P}$-images of each other, i.e., the corresponding classes $\epsilon_{w}^{P_{2}}$ are Poincaré dual to each other.

\begin{tabular}{|c|c|c|c|c|c|c|c|}
\hline$\epsilon_{w}^{P_{2}}$ & $w$ & $\lambda_{w}$ & $n_{w}$ & $\epsilon_{w}^{P_{2}}$ & $w$ & $\lambda_{w}$ & $n_{w}$ \\
\hline$b_{0}=1$ & $e$ & $(1,1,0,0)$ & 5 & $b_{9}$ & $s_{2} s_{4} s_{1} s_{2} s_{3} s_{2} s_{1} s_{4} s_{2}$ & $(-1,-1,0,0)$ & -5 \\
\hline \hline$b_{1}$ & $s_{2}$ & $(1,0,1,0)$ & 4 & $b_{8}$ & $s_{4} s_{1} s_{2} s_{3} s_{2} s_{1} s_{4} s_{2}$ & $(-1,0,-1,0)$ & -4 \\
\hline \hline$b_{2}^{1}$ & $s_{1} s_{2}$ & $(0,1,1,0)$ & 3 & $b_{7}^{1}$ & $s_{4} s_{2} s_{3} s_{2} s_{1} s_{4} s_{2}$ & $(0,-1,-1,0)$ & -3 \\
\hline$b_{2}^{2}$ & $s_{3} s_{2}$ & $(1,0,0,1)$ & 3 & $b_{7}^{2}$ & $s_{4} s_{2} s_{1} s_{2} s_{3} s_{4} s_{2}$ & $(-1,0,0,-1)$ & -3 \\
\hline$b_{2}^{3}$ & $s_{4} s_{2}$ & $(1,0,0,-1)$ & 3 & $b_{7}^{3}$ & $s_{3} s_{2} s_{1} s_{2} s_{4} s_{3} s_{2}$ & $(-1,0,0,1)$ & -3 \\
\hline \hline$b_{3}^{1}$ & $s_{3} s_{1} s_{2}$ & $(0,1,0,1)$ & 2 & $b_{6}^{1}$ & $s_{4} s_{2} s_{3} s_{1} s_{4} s_{2}$ & $(0,-1,0,-1)$ & -2 \\
\hline$b_{3}^{2}$ & $s_{4} s_{1} s_{2}$ & $(0,1,0,-1)$ & 2 & $b_{6}^{2}$ & $s_{3} s_{2} s_{4} s_{1} s_{3} s_{2}$ & $(0,-1,0,1)$ & -2 \\
\hline$b_{3}^{3}$ & $s_{4} s_{3} s_{2}$ & $(1,0,-1,0)$ & 2 & $b_{6}^{3}$ & $s_{1} s_{2} s_{3} s_{4} s_{1} s_{2}$ & $(-1,0,1,0)$ & -2 \\
\hline \hline$b_{4}^{1}$ & $s_{2} s_{3} s_{1} s_{2}$ & $(0,0,1,1)$ & 1 & $b_{5}^{1}$ & $s_{4} s_{2} s_{1} s_{3} s_{2}$ & $(0,0,-1,-1)$ & -1 \\
\hline$b_{4}^{2}$ & $s_{4} s_{3} s_{1} s_{2}(0,1,-1,0)$ & 1 & $b_{5}^{2}$ & $s_{2} s_{4} s_{1} s_{3} s_{2}$ & $(0,-1,1,0)$ & -1 \\
\hline$b_{4}^{3}$ & $s_{2} s_{4} s_{1} s_{2}(0,0,1,-1)$ & 1 & $b_{5}^{3}$ & $s_{3} s_{2} s_{1} s_{4} s_{2}$ & $(0,0,-1,1)$ & -1 \\
\hline$b_{4}^{4}$ & $s_{2} s_{4} s_{3} s_{2}$ & $(1,-1,0,0)$ & 1 & $b_{5}^{4}$ & $s_{1} s_{2} s_{3} s_{4} s_{2}$ & $(-1,1,0,0)$ & -1 \\
\hline
\end{tabular}

From the definition of $\odot_{0}$ and the values of $n_{w}$, we get the following.

Corollary 4.1. For $u, v \in W^{P_{2}}$, in $\left(H^{*}\left(G / P_{2}\right), \odot_{0}\right)$,

$$
\begin{aligned}
\epsilon_{u}^{P_{2}} \odot_{0} \epsilon_{v}^{P_{2}} & =\epsilon_{u}^{P_{2}} \cdot \epsilon_{v}^{P_{2}}, \text { if } \ell(u)+\ell(v) \leq 4 \\
& =\epsilon_{u}^{P_{2}} \cdot \epsilon_{v}^{P_{2}}, \text { if } \ell(u)+\ell(v) \geq 5 \text { and one of } \ell(u) \text { or } \ell(v) \geq 5 \\
& =0, \text { if } \ell(u)+\ell(v) \geq 5 \text { and both of } \ell(u) \text { and } \ell(v) \leq 4 .
\end{aligned}
$$

For any $i \neq j \in\{1,3,4\}$, let $\sigma_{i, j}$ be the involution of $H^{*}\left(G / P_{2}\right)$ induced from the Dynkin diagram involution taking the $i$-th node to the $j$-th node and fixing the other two nodes. Let $\hat{F}$ be the group of automorphisms of $H^{*}\left(G / P_{2}\right)$ 
generated by $\sigma_{1,3}, \sigma_{1,4}$ and $\sigma_{3,4}$. Then, $\hat{F}$ is isomorphic with the symmetric group $S_{3}$.

Using [KuLM, Lemma 2.9], the Chevalley formula and Corollary 4.1, we only need to calculate $b_{2} b_{2}^{*}, b_{2} b_{5}^{*}, b_{2} b_{6}^{*}$ and $b_{3} b_{5}^{*}$. Further, using the automorphism group $\hat{F}$, it suffices to calculate $b_{2}^{1} b_{2}^{*}, b_{2}^{1} b_{5}^{*}, b_{2}^{1} b_{6}^{*}$ and $b_{3}^{1} b_{5}^{*}$. To calculate $b_{2}^{1} b_{6}^{*}$, write

$$
b_{2}^{1} b_{6}^{*}=d b_{8}, \text { for some } d .
$$

Multiply this equation by $b_{1}$ and use the known part of the multiplication table to determine $d$. The calculation of $b_{3}^{1} b_{5}^{*}$ is exactly similar.

To calculate $b_{2}^{1} b_{5}^{2}$, write

$$
b_{2}^{1} b_{5}^{2}=\sum_{i=1}^{3} d_{i} b_{7}^{i}, \text { for some } d_{i} \in \mathbb{Z}_{+} .
$$

Multiplying the above equation by $b_{1}$, we get

$$
b_{2}^{1} b_{5}^{2} b_{1}=\sum_{i=1}^{3} d_{i} b_{8}
$$

On the other hand,

$$
b_{2}^{1} b_{5}^{2} b_{1}=\sum_{i=1}^{3} b_{2}^{1} b_{6}^{i}=2 b_{8} .
$$

Thus, $d_{1}+d_{2}+d_{3}=2$. Using the involution $\sigma_{3,4}$ of $H^{*}\left(G / P_{2}\right)$, we are forced to have

$$
b_{2}^{1} b_{5}^{2}=b_{7}^{2}+b_{7}^{3} \text { or } b_{2}^{1} b_{5}^{2}=2 b_{7}^{1} .
$$

If $b_{2}^{1} b_{5}^{2}=2 b_{7}^{1}$, then the coefficient of $b_{4}^{2}$ in $b_{2}^{1} b_{2}^{1}$ would be 2 (as can be seen by multiplying with $b_{5}^{2}$ ). Using the Chevalley formula, the coefficient of $b_{4}^{2}$ in $b_{2}^{1} \epsilon_{s_{1}} \epsilon_{s_{2}} \in H^{*}(G / B)$ can be seen to be 0 . On the other hand, $\epsilon_{s_{1}} \epsilon_{s_{2}}=b_{2}^{1}+\epsilon_{s_{2} s_{1}}$ and thus the coefficient of $b_{4}^{2}$ in $b_{2}^{1} \epsilon_{s_{1}} \epsilon_{s_{2}}$ would at least be 2 . This is a contradiction and hence we must have

$$
b_{2}^{1} b_{5}^{2}=b_{7}^{2}+b_{7}^{3} .
$$

The calculation for $b_{2}^{1} b_{5}^{4}$ is similar and yields 0 . To calculate $b_{2}^{1} b_{5}^{1}$, write

$$
b_{1} b_{1} b_{5}^{1}=\sum_{i=1}^{3} b_{2}^{i} b_{5}^{1}
$$

But,

$$
b_{2}^{3} b_{5}^{1}=\sigma_{1,4}\left(b_{2}^{1} b_{5}^{4}\right)=0
$$


and $b_{2}^{2} b_{5}^{1}=\sigma_{1,3}\left(b_{2}^{1} b_{5}^{1}\right)$. On the other hand

$$
b_{1} b_{1} b_{5}^{1}=b_{1} b_{6}^{1}=b_{7}^{1}+b_{7}^{2} .
$$

Thus,

$$
b_{2}^{1} b_{5}^{1}=b_{7}^{1} \text { or } b_{7}^{2}
$$

If $b_{2}^{1} b_{5}^{1}=b_{7}^{2}$, then

$$
b_{2}^{1} b_{5}^{3}=\sigma_{3,4}\left(b_{2}^{1} b_{5}^{1}\right)=b_{7}^{3}
$$

To calculate $b_{2}^{1} b_{2}^{*}$, write

$$
b_{2}^{1} b_{2}^{*}=\sum_{i=1}^{4} d_{i} b_{4}^{i}, \text { for some } d_{i} \in \mathbb{Z}_{+}
$$

Multiply this equation by $b_{5}^{i}$ to get

$$
b_{2}^{1} b_{2}^{*} b_{5}^{i}=d_{i} b_{9}
$$

From this we conclude that $b_{2}^{1} b_{2}^{1}=0$. However, by considering the morphism $P_{4} / B \rightarrow P S O(8) / B$, induced from the inclusion, we can easily see that $b_{2}^{1} b_{2}^{1} \neq 0$. This contradiction forces $b_{2}^{1} b_{5}^{1}=b_{7}^{1}$. Using $\sigma_{3,4}$ as above, we can calculate $b_{2}^{1} b_{5}^{3}$ from $b_{2}^{1} b_{5}^{1}$.

Now, from the known part of the multiplication table, $b_{2}^{1} b_{2}^{*}$ can be determined.

Multiplication table for $G / P_{2}$ under the product $\odot_{0}$ : 


\begin{tabular}{|c||c||c|c|c||c|c|c||c|c|c|c|}
\hline$\odot_{0}$ & $b_{1}$ & $b_{2}^{1}$ & $b_{2}^{2}$ & $b_{2}^{3}$ & $b_{3}^{1}$ & $b_{3}^{2}$ & $b_{3}^{3}$ & $b_{4}^{1}$ & $b_{4}^{2}$ & $b_{4}^{3}$ & $b_{4}^{4}$ \\
\hline \hline$b_{1}$ & $b_{2}^{1}+b_{2}^{2}+b_{2}^{3}$ & & & & & & & & & & \\
\hline \hline$b_{2}^{1}$ & $b_{3}^{1}+b_{3}^{2}$ & $b_{4}^{1}+b_{4}^{3}$ & & & & & & & & & \\
\hline$b_{2}^{2}$ & $b_{3}^{1}+b_{3}^{3}$ & $b_{4}^{2}$ & $b_{4}^{1}+b_{4}^{4}$ & & & & & & & & \\
\hline$b_{2}^{3}$ & $b_{3}^{2}+b_{3}^{3}$ & $b_{4}^{2}$ & $b_{4}^{2}$ & $b_{4}^{3}+b_{4}^{4}$ & & & & & & & \\
\hline \hline$b_{3}^{1}$ & $b_{4}^{1}+b_{4}^{2}$ & 0 & 0 & 0 & 0 & & & & & & \\
\hline$b_{3}^{2}$ & $b_{4}^{2}+b_{4}^{3}$ & 0 & 0 & 0 & 0 & 0 & & & & & \\
\hline$b_{3}^{3}$ & $b_{4}^{2}+b_{4}^{4}$ & 0 & 0 & 0 & 0 & 0 & 0 & & & & \\
\hline \hline$b_{4}^{1}$ & 0 & 0 & 0 & 0 & 0 & 0 & 0 & 0 & & & \\
\hline$b_{4}^{2}$ & 0 & 0 & 0 & 0 & 0 & 0 & 0 & 0 & 0 & & \\
\hline$b_{4}^{3}$ & 0 & 0 & 0 & 0 & 0 & 0 & 0 & 0 & 0 & 0 & \\
\hline$b_{4}^{4}$ & 0 & 0 & 0 & 0 & 0 & 0 & 0 & 0 & 0 & 0 & 0 \\
\hline \hline$b_{5}^{1}$ & $b_{6}^{1}$ & $b_{7}^{1}$ & $b_{7}^{2}$ & 0 & $b_{8}$ & 0 & 0 & $b_{9}$ & 0 & 0 & 0 \\
\hline$b_{5}^{2}$ & $b_{6}^{1}+b_{6}^{2}+b_{6}^{3}$ & $b_{7}^{2}+b_{7}^{3}$ & $b_{7}^{1}+b_{7}^{3}$ & $b_{7}^{1}+b_{7}^{2}$ & $b_{8}$ & $b_{8}$ & $b_{8}$ & 0 & $b_{9}$ & 0 & 0 \\
\hline$b_{5}^{3}$ & $b_{6}^{2}$ & $b_{7}^{1}$ & 0 & $b_{7}^{3}$ & 0 & $b_{8}$ & 0 & 0 & 0 & $b_{9}$ & 0 \\
\hline$b_{5}^{4}$ & $b_{6}^{3}$ & 0 & $b_{7}^{2}$ & $b_{7}^{3}$ & 0 & 0 & $b_{8}$ & 0 & 0 & 0 & $b_{9}$ \\
\hline \hline$b_{6}^{1}$ & $b_{7}^{1}+b_{7}^{2}$ & $b_{8}$ & $b_{8}$ & 0 & $b_{9}$ & 0 & 0 & 0 & 0 & 0 & 0 \\
\hline$b_{6}^{2}$ & $b_{7}^{1}+b_{7}^{3}$ & $b_{8}$ & 0 & $b_{8}$ & 0 & $b_{9}$ & 0 & 0 & 0 & 0 & 0 \\
\hline$b_{6}^{3}$ & $b_{7}^{2}+b_{7}^{3}$ & 0 & $b_{8}$ & $b_{8}$ & 0 & 0 & $b_{9}$ & 0 & 0 & 0 & 0 \\
\hline \hline$b_{7}^{1}$ & $b_{8}$ & $b_{9}$ & 0 & 0 & 0 & 0 & 0 & 0 & 0 & 0 & 0 \\
\hline$b_{7}^{2}$ & $b_{8}$ & 0 & $b_{9}$ & 0 & 0 & 0 & 0 & 0 & 0 & 0 & 0 \\
\hline$b_{7}^{3}$ & $b_{8}$ & 0 & 0 & $b_{9}$ & 0 & 0 & 0 & 0 & 0 & 0 & 0 \\
\hline \hline$b_{8}$ & $b_{9}$ & 0 & 0 & 0 & 0 & 0 & 0 & 0 & 0 & 0 & 0 \\
\hline
\end{tabular}

5. The Restricted triangle inequalities for $D_{4}$

Consider the basis $\left\{\epsilon_{i}^{*}\right\}_{i=1, \ldots, 4}$ of $\mathfrak{h}$ which is dual to the standard basis $\left\{\epsilon_{i}\right\}_{i=1, \ldots, 4}$ of $\mathfrak{h}^{*}$ as in [Bo, Planche IV]. Express any $h \in \mathfrak{a}$ in this basis:

$$
h=x \epsilon_{1}^{*}+y \epsilon_{2}^{*}+z \epsilon_{3}^{*}+w \epsilon_{4}^{*}, \quad x, y, z, w \in \mathbb{R} .
$$

Then,

$$
h \in \Delta \text { iff } x \geq y \geq z \geq|w| .
$$

\subsection{The restricted triangle inequalities corresponding to the parabolic} subgroup $P_{1}$. We give below the complete list (up to a permutation) of the Schubert classes $\left(b_{i_{1}}^{j_{1}}, b_{i_{2}}^{j_{2}}, b_{i_{3}}^{j_{3}}\right)=\left(\epsilon_{w_{1}}^{P_{1}}, \epsilon_{w_{2}}^{P_{1}}, \epsilon_{w_{3}}^{P_{1}}\right)$ such that

$$
\epsilon_{w_{1}}^{P_{1}} \odot_{0} \epsilon_{w_{2}}^{P_{1}} \odot_{0} \epsilon_{w_{3}}^{P_{1}}=\epsilon_{w_{o}^{P_{1}}}^{P_{1}}
$$


and write down the corresponding inequality $T_{\mathbf{w}}^{P_{1}}$ :

$$
\sum_{j=1}^{3}\left\langle\lambda_{w_{j}}^{P_{1}}, h_{j}\right\rangle \geq 0 .
$$

We express $h_{j}=\left(x_{j}, y_{j}, z_{j}, w_{j}\right), j=1,2,3$ in the coordinates $\left\{\epsilon_{i}^{*}\right\}_{i=1, \ldots, 4}$. We divide the set of inequalities in two disjoint sets, one coming from the Schubert classes $\left(b_{i_{1}}^{j_{1}}, b_{i_{2}}^{j_{2}}, b_{i_{3}}^{j_{3}}\right)$ such that at least one of the cohomology classes is 1 . It can be seen that the corresponding inequalities are the weak triangle inequalities WTI defined in Section 3.1.1. The remaining inequalities are called the essential triangle inequalities ETI. We label the inequalities ETI corresponding to the parabolic $P_{1}$ by $E T I(1)$ and similarly for $W T I$.

\section{$\operatorname{ETI}(1):$}

WTI(1):

$$
\begin{array}{ll}
\left(b_{1}, b_{1}, b_{4}\right): & y_{1}+y_{2}-z_{3} \geq 0 \\
\left(b_{1}, b_{2}, b_{3}^{1}\right): & y_{1}+z_{2}+w_{3} \geq 0 \\
\left(b_{2}, b_{3}^{2}, b_{1}\right): & z_{1}-w_{2}+y_{3} \geq 0
\end{array}
$$

$$
\begin{array}{cc}
\left(1,1, b_{6}\right): & x_{1}+x_{2}-x_{3} \geq 0 \\
\left(1, b_{1}, b_{5}\right): & x_{1}+y_{2}-y_{3} \geq 0 \\
\left(1, b_{2}, b_{4}\right): & x_{1}+z_{2}-z_{3} \geq 0 \\
\left(1, b_{3}^{1}, b_{3}^{2}\right): & x_{1}+w_{2}-w_{3} \geq 0
\end{array}
$$

To get the full set of inequalities $T_{\mathbf{w}}^{P_{1}}$ for $P_{1}$, we need to permute the above collection of inequalities where the subscripts $\{1,2,3\}$ are permuted arbitrarily. The number at the end of each inequality denotes the number of inequalities obtained by permuting that particular inequality. Thus, the total number of inequalities $T_{\mathbf{w}}^{P_{1}}$ corresponding to $P_{1}$ is 36 .

\subsection{The restricted triangle inequalities $T_{\mathrm{w}}^{P_{2}}$ corresponding to the par-} abolic subgroup $P_{2}$. In each cohomological degree except for 8 and 10 there is only one orbit of the Schubert classes under $\hat{F}$. In degree 8 there are two orbits (of three classes in the orbit of $b_{4}^{1}$ and one in the orbit of $b_{4}^{2}$ ).

Of course, $\hat{F}$ acts diagonally on the set of triples $\left(b_{i_{1}}^{j_{1}}, b_{i_{2}}^{j_{2}}, b_{i_{3}}^{j_{3}}\right)$ such that $b_{i_{1}}^{j_{1}} \odot_{0}$ $b_{i_{2}}^{j_{2}} \odot_{0} b_{i_{3}}^{j_{3}}=b_{9}$. Also, $S_{3}$ acts on such triples via permutation and these two actions commute. So, we get an action of the product group $S_{3} \times \hat{F}$ on such triples. The following is a complete list of such triples of Schubert classes in $H^{*}\left(G / P_{2}\right)$ up to 
the action of $S_{3} \times \hat{F}$ and the corresponding inequality $T_{\mathbf{w}}^{P_{2}}$. The number at the end of each inequality denotes the order of the corresponding $S_{3} \times \hat{F}$-orbit.

\section{$\operatorname{ETI}(2):$}

$$
\begin{array}{ll}
\left(b_{1}, b_{1}, b_{7}^{1}\right): & x_{1}+z_{1}+x_{2}+z_{2}-y_{3}-z_{3} \geq 0 \\
\left(b_{1}, b_{2}^{1}, b_{6}^{1}\right): & x_{1}+z_{1}+y_{2}+z_{2}-y_{3}-w_{3} \geq 0 \\
\left(b_{1}, b_{3}^{1}, b_{5}^{1}\right): & x_{1}+z_{1}+y_{2}+w_{2}-z_{3}-w_{3} \geq 0 \\
\left(b_{1}, b_{3}^{1}, b_{5}^{2}\right): & x_{1}+z_{1}+y_{2}+w_{2}-y_{3}+z_{3} \geq 0 \\
\left(b_{2}^{1}, b_{2}^{1}, b_{5}^{1}\right): & y_{1}+z_{1}+y_{2}+z_{2}-z_{3}-w_{3} \geq 0 \\
\left(b_{2}^{1}, b_{2}^{2}, b_{5}^{2}\right): & y_{1}+z_{1}+x_{2}+w_{2}-y_{3}+z_{3} \geq 0
\end{array}
$$

\section{WTI(2):}

$$
\begin{array}{cc}
\left(1,1, b_{9}\right): & x_{1}+y_{1}+x_{2}+y_{2}-x_{3}-y_{3} \geq 0 \\
\left(b_{1}, 1, b_{8}\right): & x_{1}+z_{1}+x_{2}+y_{2}-x_{3}-z_{3} \geq 0 \\
\left(b_{2}^{1}, 1, b_{7}^{1}\right): & y_{1}+z_{1}+x_{2}+y_{2}-y_{3}-z_{3} \geq 0 \\
\left(b_{3}^{1}, 1, b_{6}^{1}\right): & y_{1}+w_{1}+x_{2}+y_{2}-y_{3}-w_{3} \geq 0 \\
\left(b_{4}^{1}, 1, b_{5}^{1}\right): & z_{1}+w_{1}+x_{2}+y_{2}-z_{3}-w_{3} \geq 0 \\
\left(b_{4}^{2}, 1, b_{5}^{2}\right): & y_{1}-z_{1}+x_{2}+y_{2}-y_{3}+z_{3} \geq 0
\end{array}
$$

The group $S_{3} \times F$ acts canonically on $\mathfrak{a}^{3}$, where $S_{3}$ acts by permutation of the three factors and $F$ acts via the corresponding Dynkin automorphism of $\mathfrak{a}$. To get the full set of inequalities $T_{\mathbf{w}}^{P_{2}}$ for $P_{2}$, we need to apply the group $S_{3} \times F$ to the above collection of inequalities.

Thus, we get totally 186 inequalities corresponding to the maximal parabolic $P_{2}$.

The multiplication table for $H^{*}\left(G / P_{3}\right)$ (resp. $H^{*}\left(G / P_{4}\right)$ ) can be obtained from that of $H^{*}\left(G / P_{1}\right)$ by using the isomorphism of $H^{*}\left(G / P_{1}\right)$ with $H^{*}\left(G / P_{3}\right)$ (resp. $\left.H^{*}\left(G / P_{4}\right)\right)$ induced from the Dynkin automorphisms. Accordingly, the inequalities corresponding to $H^{*}\left(G / P_{3}\right)$ and $H^{*}\left(G / P_{4}\right)$ are obtained from $T_{\mathbf{w}}^{P_{1}}$ by applying the action of $F$. All in all, each system $T_{\mathbf{w}}^{P_{3}}$ and $T_{\mathbf{w}}^{P_{4}}$ consists of 36 inequalities.

Below are the explicit lists of inequalities. 
5.3. The restricted triangle inequalities corresponding to the parabolic subgroup $P_{3}$.

\section{$\operatorname{ETI}(3)$ :}

$$
\begin{aligned}
& x_{1}+y_{1}-z_{1}+w_{1}+x_{2}+y_{2}-z_{2}+w_{2}-x_{3}+y_{3}-z_{3}-w_{3} \geq 0 \\
& x_{1}+y_{1}-z_{1}+w_{1}+x_{2}-y_{2}+z_{2}+w_{2}-x_{3}+y_{3}+z_{3}+w_{3} \geq 0 \\
& x_{1}-y_{1}+z_{1}+w_{1}+x_{2}-y_{2}-z_{2}-w_{2}+x_{3}+y_{3}-z_{3}+w_{3} \geq 0
\end{aligned}
$$

WTI(3):

$$
\begin{aligned}
& x_{1}+y_{1}+z_{1}-w_{1}+x_{2}+y_{2}+z_{2}-w_{2}-x_{3}-y_{3}-z_{3}+w_{3} \geq 0 \\
& x_{1}+y_{1}+z_{1}-w_{1}+x_{2}+y_{2}-z_{2}+w_{2}-x_{3}-y_{3}+z_{3}-w_{3} \geq 0 \\
& x_{1}+y_{1}+z_{1}-w_{1}+x_{2}-y_{2}+z_{2}+w_{2}-x_{3}+y_{3}-z_{3}-w_{3} \geq 0 \\
& x_{1}+y_{1}+z_{1}-w_{1}-x_{2}+y_{2}+z_{2}+w_{2}+x_{3}-y_{3}-z_{3}-w_{3} \geq 0
\end{aligned}
$$

To get the full set of inequalities $T_{\mathbf{w}}^{P_{3}}$ for $P_{3}$, we need to permute the above collection of inequalities where the subscripts $\{1,2,3\}$ are permuted arbitrarily.

\subsection{The restricted triangle inequalities corresponding to the parabolic} subgroup $P_{4}$.

$\operatorname{ETI}(4)$ :

$$
\begin{aligned}
& x_{1}+y_{1}-z_{1}-w_{1}+x_{2}+y_{2}-z_{2}-w_{2}-x_{3}+y_{3}-z_{3}+w_{3} \geq 0 \\
& x_{1}+y_{1}-z_{1}-w_{1}+x_{2}-y_{2}+z_{2}-w_{2}+x_{3}-y_{3}-z_{3}+w_{3} \geq 0 \\
& x_{1}-y_{1}+z_{1}-w_{1}-x_{2}+y_{2}+z_{2}-w_{2}+x_{3}+y_{3}-z_{3}-w_{3} \geq 0
\end{aligned}
$$

\section{WTI(4):}

$$
\begin{aligned}
& x_{1}+y_{1}+z_{1}+w_{1}+x_{2}+y_{2}+z_{2}+w_{2}-x_{3}-y_{3}-z_{3}-w_{3} \geq 0 \\
& x_{1}+y_{1}+z_{1}+w_{1}+x_{2}+y_{2}-z_{2}-w_{2}-x_{3}-y_{3}+z_{3}+w_{3} \geq 0 \\
& x_{1}+y_{1}+z_{1}+w_{1}+x_{2}-y_{2}+z_{2}-w_{2}-x_{3}+y_{3}-z_{3}+w_{3} \geq 0 \\
& x_{1}+y_{1}+z_{1}+w_{1}+x_{2}-y_{2}-z_{2}+w_{2}-x_{3}+y_{3}+z_{3}-w_{3} \geq 0
\end{aligned}
$$

To get the full set of inequalities $T_{\mathbf{w}}^{P_{4}}$ for $P_{4}$, we need to permute the above collection of inequalities where the subscripts $\{1,2,3\}$ are permuted arbitrarily. 
5.5. The cone $\mathcal{C}$. Thus, the total number of inequalities $T_{\mathbf{w}}^{P_{i}}$ defining the cone $\mathcal{C}$ inside $\Delta^{3}$ is equal to $36+186+36+36=294$. Since $\Delta^{3} \subset \mathfrak{a}^{3}$ is defined by 12 inequalities, we get altogether 306 inequalities defining the cone $\mathcal{C}$ inside $\mathfrak{a}^{3}$. Let $\Sigma$ be the set of these 306 inequalities defining the cone $\mathcal{C}$.

Theorem 5.1. The system $\Sigma$ is irredundant.

Proof. In order to show the irredundancy of the system $\Sigma$, it suffices to show that the cone $\mathcal{C}$ has 306 facets. It is done by applying the MAPLE package CONVEX $[\mathrm{F}]$ to the above system (see $[\mathrm{Ka} 1]$ ).

Remark 5.2. The same computation also shows that the cone $\mathcal{C}$ has 81 extremal rays.

Our next goal is to verify the saturation conjecture (Conjecture 1.5) for the group $G^{\vee}=\operatorname{Spin}(8)$. Let $P^{\vee} \subset\left(\mathfrak{h}^{\vee}\right)^{*}=\mathfrak{h}$ denote the weight lattice of $G^{\vee}$ and $Q^{\vee} \subset P^{\vee}$ denote the root lattice. Of course, $Q=Q^{\vee}$ since $G$ is simply-laced. Recall that in the Introduction we have defined the semigroups $\mathcal{C}^{0}$ and $\mathcal{R}$ of $\mathcal{D}^{3}$ with

$$
\mathcal{R} \subset \mathcal{C}^{0}
$$

Theorem 5.3 (Saturation theorem for $\operatorname{Spin}(8)$ ).

$$
\mathcal{R}=\mathcal{C}^{0} .
$$

Proof. In order to prove the inclusion $\mathcal{C}^{0} \subset \mathcal{R}$, it suffices to show that each semigroup generator of $\mathcal{C}^{0}$ belongs to $\mathcal{R}$. To find the minimal set of semigroup generators (Hilbert basis) for $\mathcal{C}^{0}$, we define a basis $\left\{\bar{\alpha}_{i}, \zeta_{j}\right\}_{1 \leq i \leq 4,1 \leq j \leq 8}$ of the lattice $\phi^{-1}\left(Q^{\vee}\right)$, where

$$
\phi:\left(P^{\vee}\right)^{3} \rightarrow P^{\vee}, \phi(\lambda, \mu, \nu)=\lambda+\mu+\nu .
$$

Consider the splitting of the exact sequence (for $K:=\operatorname{Ker} \phi$ )

$$
0 \rightarrow K \rightarrow\left(P^{\vee}\right)^{3} \stackrel{\phi}{\rightarrow} P^{\vee} \rightarrow 0
$$

over $Q^{\vee}$ under the map $\psi\left(\alpha_{i}\right)=\left(\alpha_{i}, 0,0\right), i=1, \ldots, 4$.

Therefore, we can identify $Q^{\vee}$ with the subgroup $\psi\left(Q^{\vee}\right) \subset\left(P^{\vee}\right)^{3}$ with basis

$$
\left\{\bar{\alpha}_{i}=\psi\left(\alpha_{i}\right)\right\}_{1 \leq i \leq 4} .
$$


Set

$$
\zeta_{j}=\left(-\omega_{j}, \omega_{j}, 0\right)
$$

for $1 \leq j \leq 4$ and

$$
\zeta_{j}=\left(-\omega_{j-4}, 0, \omega_{j-4}\right)
$$

for $5 \leq j \leq 8$, where $\left\{\omega_{j}\right\}$ are the fundamental weights for $\operatorname{Spin}(8)$. Then, it is clear that $\left\{\zeta_{j}, j=1, \ldots, 8\right\}$ is a basis of $K$ and

$$
\left\{\bar{\alpha}_{i}, \zeta_{j}: i=1, \ldots, 4, j=1, \ldots, 8\right\}
$$

is a basis of $\phi^{-1}\left(Q^{\vee}\right)$.

Thus, the semigroup $\mathcal{C}^{0}$ is precisely equal to the integral points of the cone $\mathcal{C}$ with respect to the coordinates $\left\{\bar{\alpha}_{i}, \zeta_{j}\right\}$.

Computation of the Hilbert basis $H$ of $\mathcal{C}^{0}$ is done via the package HILBERT, [HHM] (see [Ka2]). Observe that the action of the group $S_{3} \times F$ on $\mathfrak{a}^{3}$ keeps $\mathcal{C}^{0}$ stable. Since the Hilbert basis is unique, it follows that $H$ is invariant under the action of $S_{3} \times F$. Below is the list $H^{\prime}$ of elements of $H$ modulo the action of $S_{3} \times F$ :

$$
\begin{gathered}
\left(\omega_{1}, \omega_{1}, 0\right) \\
\left(\omega_{2}, \omega_{2}, 0\right) \\
\left(\omega_{2}, \omega_{2}, \omega_{2}\right) \\
\left(\omega_{1}, \omega_{3}, \omega_{4}\right) \\
\left(\omega_{1}, \omega_{1}, \omega_{2}\right) \\
\left(\omega_{1}, \omega_{2}, \omega_{3}+\omega_{4}\right) \\
\left(2 \omega_{1}, \omega_{2}, \omega_{2}\right) \\
\left(\omega_{1}+\omega_{2}, \omega_{2}, \omega_{3}+\omega_{4}\right) \\
\left(\omega_{2}, \omega_{2}, \omega_{1}+\omega_{3}+\omega_{4}\right) \\
\left(2 \omega_{2}, \omega_{2}, \omega_{1}+\omega_{3}+\omega_{4}\right) .
\end{gathered}
$$

Since $S_{3} \times F$ also preserves the semigroup $\mathcal{R}$, in order to prove Theorem 5.3, it suffices to check that $H^{\prime} \subset \mathcal{R}$. This is done using MAPLE package WEYL written by John Stembridge, see [S]. It is done in [Ka2].

\section{REFERENCES}

[AMW] A. Alekseev, E. Meinrenken and C. Woodward, Linearization of Poisson actions and singular values of matrix products, Ann. Inst. Four., vol. 51 (2001), 1691-1717. 
[B] P. Belkale, Geometric proofs of Horn and saturation conjectures, J. Alg. Geom., vol. 15 (2006), 133-173.

[BK] P. Belkale and S. Kumar, Eigenvalue problem and a new product in cohomology of flag varietes, Inventiones Math., vol. 166 (2006), 185-228.

[BS] A. Berenstein and R. Sjamaar, Coadjoint orbits, moment polytopes, and the HilbertMumford criterion, J. Amer. Math. Soc., vol. 13 (2000), 433-466.

[Bo] N. Bourbaki, "Groupes et Algèbres de Lie", Chap. 4-6, Masson, Paris, 1981.

[DW] H. Derksen and J. Weyman, Semi-invariants of quivers and saturation for LittlewoodRichardson coefficients, J. Amer. Math. Soc., vol. 13 (2000), 467-479.

[EL] S. Evens and J.-H. Lu, Thompson's conjecture for real semi-simple Lie groups, In: "The breadth of symplectic and Poisson geometry," Festschrift in honor of Alan Weinstein, Birkhäuser, Progress in Math., vol. 232 (2005), 121-137.

[F] M. Franz, Convex - a Maple package for convex geometry, 2006. Available at http://www-fourier.ujf-grenoble.fr/ franz/convex

[He] S. Helgason, "Differential Geometry, Lie groups, and Symmetric Spaces", Academic Press, 1978 .

[HHM] R. Hemmecke, R. Hemmecke and P. Malkin, 4ti2, Version 1.2-Computation of Hilbert bases, Graver bases, toric Gröbner bases, and more, 2005.

Available at http://www.4ti2.de

[Ka1] M. Kapovich, Proof of irredundancy of Belkale-Kumar stability inequalities for PO(8). Available at http://www.math.ucdavis.edu/ kapovich/EPR/irred.mw

[Ka2] M. Kapovich, Proof of Saturation Theorem for the tensor products of irreducible representations of $\operatorname{Spin}(8)$.

Available at http://www.math.ucdavis.edu/ kapovich/EPR/hbasis.mw

[KLM1] M. Kapovich, B. Leeb and J. J. Millson, Convex functions on symmetric spaces, side lengths of polygons and the stability inequalities for weighted configurations at infinity, Journal of Differential Geometry, to appear.

[KLM2] M. Kapovich, B. Leeb and J. J. Millson, Polygons in buildings and their refined sidelengths, Preprint, 2004, arXiv:math.MG/0406305.

[KLM3] M. Kapovich, B. Leeb and J. J. Millson, The generalized triangle inequalities in symmetric spaces and buildings with applications to algebra, Memoirs of AMS, vol. 192, 2008.

[KM1] M. Kapovich and J. J. Millson, A path model for geodesics in Euclidean buildings and its applications to representation theory, Groups, Geometry and Dynamics, vol. 2 (2008), 405-480.

[KM2] M. Kapovich and J. J. Millson, Structure of the tensor product semigroup, Asian J. of Math., vol. 10 (2006), 492-540.

[K11] A. Klyachko, Stable bundles, representation theory and Hermitian operators, Selecta Mathematica, vol. 4 (1998), 419-445.

[K12] A. Klyachko, Random walks on symmetric spaces and inequalities for matrix spectra, Linear Algebra Appl., vol. 319 (2000), 37-59. 
$[\mathrm{KT}]$ A. Knutson and T. Tao, The honeycomb model of $G L_{n}(\mathbb{C})$ tensor products I: Proof of the saturation conjecture, J. Amer. Math. Soc., vol. 12 (1999), 1055-1090.

[KTW] A. Knutson, T. Tao and C. Woodward, The honeycomb model of $G L_{n}(\mathbb{C})$ tensor products II: Puzzles determine facets of the Littlewood-Richardson cone, J. Amer. Math. Soc., vol. 17 (2004), 19-48.

$[\mathrm{K}] \quad$ S. Kumar, "Kac-Moody groups, their flag varieties and representation theory", Birkhäuser, Progress in Mathematics, vol. 204, 2002.

[KuLM] S. Kumar, B. Leeb and J. J. Millson, The generalized triangle inequalities for rank 3 symmetric spaces of noncompact type, Contemp. Math., vol. 332 (2003), 171-195.

[R] N. Ressayre, Geometric invariant theory and generalized eigenvalue problem, Preprint (2007).

[Sp] T. A. Springer, Reductive groups, In: "Automorphic forms, representations, and Lfunctions," Proc. Symp. AMS, vol. 33 (1979), 3-27.

[S] J. Stembridge, Coxeter and Weyl Maple packages.

Available at http://www.math.lsa.umich.edu/ jrs/maple.html

Michael Kapovich

Department of Mathematics, University of California

Davis, CA 95616, USA

E-mail: kapovich@math.ucdavis.edu

Shrawan Kumar

Department of Mathematics, University of North Carolina

Chapel Hill, NC 27599-3250, USA

E-mail: shrawan@email.unc.edu

John J. Millson

Department of Mathematics, University of Maryland

College Park, MD 20742, USA

E-mail: jjm@math.umd.edu 